\title{
GSK-3 Inhibitors: Anti-Diabetic Treatment Associated with Cardiac Risk?
}

\author{
Editorial to: "The Impact of Chronic Glycogen Synthase Kinase-3 Inhibition on Remodeling \\ of Normal and Pre-Diabetic Rat Hearts." by Barbara Huisamen et al.
}

\author{
Miranda Nabben $^{1} \cdot$ Dietbert Neumann $^{1}$
}

Published online: 16 June 2016

(C) Springer Science+Business Media New York 2016

The therapeutic potential of glycogen synthase kinase-3 (GSK-3) inhibitors for treatment in diabetes was first recognized in the late 1990s. GSK-3 is a serine/threonine kinase that phosphorylates, and thereby, regulates the function of many metabolic, signaling and structural proteins. GSK-3 is constitutively active in the basal state of cells and is inhibited in response to a variety of hormones, growth factors and other mediators as a result of phosphorylation (Ser21 in the $\alpha$ isoform and Ser9 in the $\beta$-isoform). Particularly, the functional role of GSK-3 in insulin signaling and glucose metabolism makes it a particularly intriguing candidate target for treatment of type 2 diabetes. Activated GSK-3 phosphorylates and thereby inactivates glycogen synthase, an enzyme involved in converting glucose to glycogen for storage. Insulin can relieve GSK-3-mediated inhibition on glycogen synthase by binding to its receptor and activating the PI3K / Akt signaling pathway. In adipose tissue of insulin-resistant obese rodent models [1] and skeletal muscle of obese type 2 diabetic patients [2] the activity and expression of GSK-3 have been reported to be elevated. Overexpression of human GSK-3 in skeletal muscle of mice results in impaired glucose tolerance and suppressed glycogen synthase activity and glycogen synthesis, further supporting a role for GSK-3 in type 2 diabetes [3]. Furthermore, overexpression of GSK-3 has been shown to attenuate insulin signaling due to phosphorylation and downregulation of insulin receptor substrate-1 [reviewed in [4]]. Therefore, it has been suggested that drugs inhibiting GSK-3

Miranda Nabben

m.nabben@maastrichtuniversity.nl

1 Department of Genetics \& Cell Biology, CARIM School for Cardiovascular Diseases, Maastricht University, P.O. Box 616, 6200, MD Maastricht, the Netherlands could mimic the ability of insulin to promote the conversion of glucose to glycogen, overcoming the resistance to insulin. Indeed, several studies with pre-diabetic and diabetic rodent models have shown that administration of GSK-3 inhibitors improved glucose tolerance and insulin sensitivity [reviewed in [5]]. These improvements were accompanied with elevated glycogen synthase activity in muscle and liver and increased liver glycogen accumulation.

On the other hand, next to its role in glucose metabolism, GSK-3 has also been recognized to play central roles in various other key biological processes, including cell growth, protein synthesis, cell differentiation, apoptosis and embryonic development (reviewed in $[6,7])$. Treatment with GSK-3 inhibitors may therefore be challenging and result in adverse side effects. With respect to the heart, several studies implicated GSK-3 in pathological processes [reviewed in [8]]. In particular, active (nonphosphorylated) GSK-3 is a potent inhibitor of cardiac hypertrophy. Consistent with this concept, homozygous GSK- $3 \alpha$ knockout mice develop cardiac hypertrophy and contractile dysfunction two months after birth [9]. Homozygous GSK-3 $\beta$ knockout mice are embryonic lethal $[7,10,11]$ and adult cardiomyocytespecific conditional GSK-3 $\beta$ knockout mice show increased cardiomyocyte proliferation [12]. Furthermore, deletion of GSK- $3 \beta$ in embryonic stem cells increases cardiomyocyte proliferation and inhibits differentiation of embryonic stem cells into cardiomyocytes $[11,13]$. This raises the question whether GSK3 inhibition causes hypertrophy in the insulin resistant condition, a condition in which GSK-3 activity is supposedly elevated. Figure 1 summarizes the expected effects of GSK-3 inhibition on cardiac hypertrophy in the normal and insulin resistant state.

In the current study, Huisamen et al. investigated the effects of 8-week treatment with the non-selective GSK-3 $\alpha / \beta$ inhibitor CHIR118637 on the cardiac hypertrophic growth in dietinduced obese (DIO) pre-diabetic rats [14]. First, the present work confirms that GSK-3 inhibition in chow-fed animals 


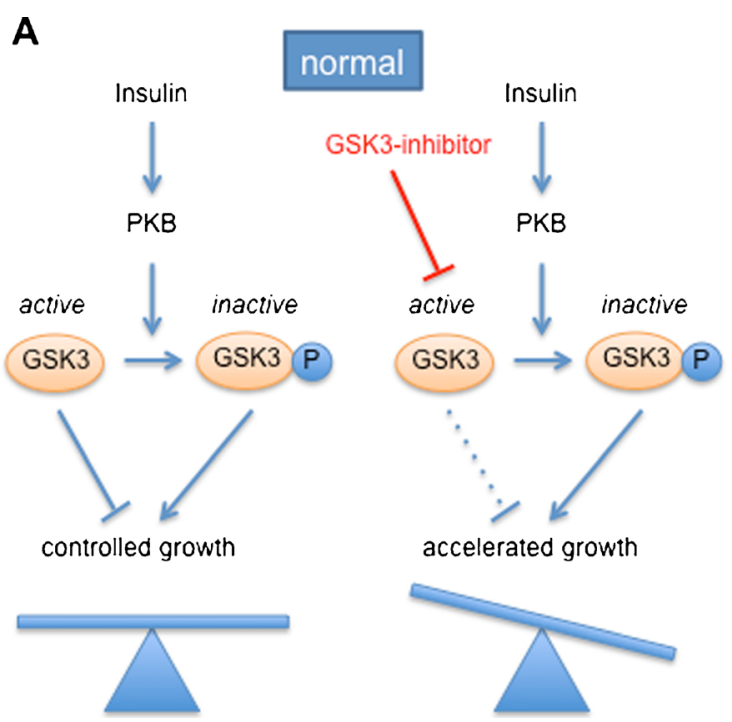

Fig. 1 GSK-3 inhibition in the context of cardiac hypertrophy. Active (non-phosphorylated) GSK-3 strongly inhibits cell growth. Insulin activates PKB, which phosphorylates and inactivates GSK-3. a In the normal 'healthy' heart, cardiac growth is regulated by the balance between active and inactive GSK-3. The treatment with GSK-3 inhibitors turns off the growth inhibitory function of GSK-3, therefore leading to accelerated growth. b In the insulin resistant state, stimulation

causes cardiac hypertrophy. Second, the study shows that obesity elicited myocardial hypertrophy (as evidenced by increased ventricular mass, enlarged cardiomyocytes and increased end diastolic diameter). Such high fat diet-induced cardiac hypertrophy recently has been described after only 6 weeks on high fat diet, i.e. before the onset of obesity [15]. Third, whereas GSK-3 inhibition led to increased enddiastolic diameter and peri-nuclear localization of the transcription factors nuclear factor of activated T-cell-c3 (NFATc3) and GATA4 in both control and DIO rats, the treatment with the GSK-3 inhibitor did not further increase ventricular mass and cardiomyocyte cell size. Since nuclear localization of NFATc3 and GATA4 that results from GSK-3 inactivation initiates the transcription of genes implicated in the development of myocardial hypertrophy, other obesity-induced signaling mechanisms likely interfere with the effects of CHIR118637, such as inflammatory signaling [15]. In addition, it has previously been discussed that GSK-3, depending on the stimulus, could be regulated independently of Akt/PKB [16].

Huisamen et al. [14] conclude that it remains unresolved whether the observed hypertrophic changes with GSK-3 inhibition are adaptive or maladaptive based upon the lack of change in echocardiographic assessment of myocardial infarction (i.e. fractional shortening) measured by echocardiography. Therefore, the current study adds data to the discussion, but cannot resolve the controversy of GSK-3 inhibition as a safe treatment strategy for obese and (pre-)diabetic humans. Further research on the precise role of GSK-3 in cardiac

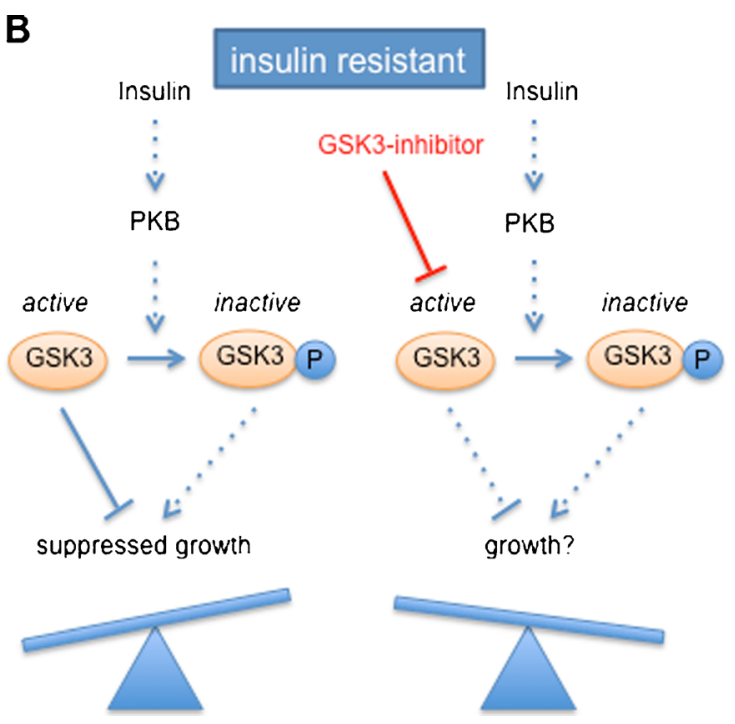

of PKB by insulin is impaired. Thus, GSK-3 expectedly would be found in a more active, i.e. growth suppressive state. Upon pharmaceutical GSK-3 inhibition in the insulin resistant condition, the output of this growth regulatory switch is unknown. Given that PKB is not totally inactive even in the insulin resistant state, it could be predicted that the growth rate rather accelerates

metabolism and function will give important new insights on the link between obesity, diabetes and hypertrophy.

Acknowledgments MN and DN are recipients of VENI- and VIDIInnovational Research Grants from the Netherlands Organization of Scientific Research (NWO-ZonMw Grant no. 916.14.050 and NWOALW Grant no. 864.10.007, respectively).

\section{References}

1. Eldar-Finkelman H, Schreyer SA, Shinohara MM, LeBoeuf RC, Krebs EG. Increased glycogen synthase kinase-3 activity in diabetes- and obesity-prone C57BL/6 J mice. Diabetes. 1999;48(8):1662-6.

2. Nikoulina SE, Ciaraldi TP, Carter L, Mudaliar S, Park KS, Henry RR. Impaired muscle glycogen synthase in type 2 diabetes is associated with diminished phosphatidylinositol 3-kinase activation. J Clin Endocrinol Metab. 2001;86(9):4307-14.

3. Pearce NJ, Arch JR, Clapham JC, Coghlan MP, Corcoran SL, Lister $\mathrm{CA}$, et al. Development of glucose intolerance in male transgenic mice overexpressing human glycogen synthase kinase-3beta on a muscle-specific promoter. Metabolism. 2004;53(10):1322-30.

4. Cohen P, Goedert M. GSK3 inhibitors: development and therapeutic potential. Nat Rev Drug Discov. 2004;3(6):479-87.

5. MacAulay K, Woodgett JR. Targeting glycogen synthase kinase-3 (GSK-3) in the treatment of type 2 diabetes. Expert Opin Ther Targets. 2008;12(10):1265-74.

6. Cohen P, Frame S. The renaissance of GSK3. Nat Rev Mol Cell Biol. 2001;2(10):769-76.

7. Kaidanovich-Beilin O, Woodgett JR. GSK-3: functional insights from cell biology and animal models. Front Mol Neurosci. 2011;4:40. 
8. Cheng H, Woodgett J, Maamari M, Force T. Targeting GSK-3 family members in the heart: a very sharp double-edged sword. J Mol Cell Cardiol. 2011;51(4):607-13.

9. Zhou J, Lal H, Chen X, Shang X, Song J, Li Y, et al. GSK-3alpha directly regulates beta-adrenergic signaling and the response of the heart to hemodynamic stress in mice. J Clin Invest. 2010;120(7): 2280-91.

10. Hoeflich KP, Luo J, Rubie EA, Tsao MS, Jin O, Woodgett JR. Requirement for glycogen synthase kinase-3beta in cell survival and NF-kappaB activation. Nature. 2000;406(6791):8690.

11. Kerkela R, Kockeritz L, Macaulay K, Zhou J, Doble BW, Beahm C, et al. Deletion of GSK-3beta in mice leads to hypertrophic cardiomyopathy secondary to cardiomyoblast hyperproliferation. J Clin Invest. 2008;118(11):3609-18.

12. Woulfe KC, Gao E, Lal H, Harris D, Fan Q, Vagnozzi R, et al. Glycogen synthase kinase-3beta regulates post-myocardial infarction remodeling and stress-induced cardiomyocyte proliferation in vivo. Circ Res. 2010;106(10):1635-45.

13. Force T, Woodgett JR. Unique and overlapping functions of GSK-3 isoforms in cell differentiation and proliferation and cardiovascular development. J Biol Chem. 2009;284(15):9643-7.

14. Huisamen B, Lubelwana-Hafver T, Lumkwana D, Lochner A. The impact of chronic glycogen synthase kinase-3 inhibition on remodeling of normal and pre-diabetic rat hearts. Cardiovasc Drugs Ther 2016;30.This issue

15. Goncalves N, Silva AF, Rodrigues PG, Correia E, Moura C, Eloy $\mathrm{C}$, et al. Early cardiac changes induced by a hypercaloric westerntype diet in "subclinical" obesity. Am J Physiol Heart Circ Physiol. 2016;310(6):H655-66.

16. Sugden PH, Fuller SJ, Weiss SC, Clerk A. Glycogen synthase kinase 3 (GSK3) in the heart: a point of integration in hypertrophic signalling and a therapeutic target? A critical analysis. Br J Pharmacol. 2008;153(Suppl 1):S137-53. 